\title{
A model for estimating spinal cord injury prevalence in the United States
}

\author{
JE Lasfargues, D Custis, F Morrone, J Carswell and T Nguyen \\ Paralyzed Veterans of America, Health Policy Department, 801 18th Street, NW, Washington, DC 20006, USA
}

\begin{abstract}
A model was developed to provide a tool to forecast demographic trends in populations of people with traumatic spinal cord injury at the national and state level. This information is critical to planning for the allocation and distribution of resources to care for people with spinal cord injury. The literature on incidence, mortality, and prevalence of spinal cord injury in the United States was reviewed and reported values were evaluated for incorporation into the model. A linear relationship between age specific survival rates of the spinal cord injury population, and expected survival rates in the absence of spinal cord injury was established and this provided the basis for projections using age cohort survival methodology. The model's projections indicate a need for future expansion of capacity to treat traumatic spinal cord injury in the private sector, and a need to prepare for an aging disabled population. The annual number of traumatic spinal cord injury cases admitted to hospitals is projected to increase from approximately 11500 in 1994 to almost 13400 in 2010. Age adjusted post-hospitalization incidence rate in 1994 is estimated at approximately 38 per million ( 23 per million for females and 55 per million for males). A $20 \%$ increase in the US spinal cord injury prevalence can be expected over the next 10 years, going from approximately 207000 estimated in 1994, to 247000 . During this time, the veteran segment, which currently comprises $22 \%$ of the spinal cord injury population, is projected to decline. Increases in the number of people aged 65 or more with spinal cord injury, currently estimated to be around $11 \%$ of the total spinal cord injury population, can be expected to grow more than $24 \%$ by 2025 to almost 73000 .
\end{abstract}

Keywords: spinal cord injury; incidence; prevalence; mortality; epidemiology

\section{Introduction}

The current environment of health care reform in the United States has created a new awareness of the need for measuring outcomes and developing optimal protocols for the care of traumatic spinal cord injury (SCI). Propelling this movement is the increased emphasis on efficient allocation of resources and the reduction of overall costs of providing care. Unfortunately, gaps in our understanding of the epidemiology of SCI have inhibited development of a credible population forecasting model that could assist in planning decisions.

Recent progress toward focusing estimates of the prevalence of traumatic spinal cord injury in the United States, along with research that has enhanced the understanding of the long term effects of SCI, particularly of life expectancy, has provided the basis for development of a population based forecasting model. The purpose of this study was to bring together what is currently known of the major components that determine SCI prevalence, into a cohesive model for estimating the size and age characteristics of not only the existing SCI population, but also that of future populations.

Prevalence of SCI is a function not only of incidence rate and survival rate, but also of the size and demographic composition of the population pool at risk. Given the baseline prevalence, the interaction of these variables determines future SCI population size. Because the values are age specific, shifting patterns of age distribution within the relevant populations translate to a prevalence rate that is dynamic over time. It is also sensitive to changes in gender distribution and injury class (neurological level and extent of lesion) case mix that affect one or all of the variables ${ }^{1}$ that determine prevalence. A linear relationship between age specific survival rates of the SCI population and expected survival rates in the absence of SCI was established that provided the basis for projections using age cohort survival methodology.

\section{Methods}

For modeling purposes the SCI population was treated as having three distinct segments (male, female and veteran) with the veteran population being a subset of the male population. Each of these populations has a unique combination of incidence rate, survival rate, and baseline prevalence. Once these critical input parameters were ascertained using data derived from studies reported in the literature, a basic model was developed and independently applied to each population. 
Age cohort survival methodology was used to make projected estimates of each of the three SCI population segments. The relationship between variables within individual age groups is represented by the following equation:

$$
P_{x+1, a}=\left[\left(P_{x, a}+I_{x, a}\right) \times S R_{a}\right]-\left(\frac{P_{x, a}}{N_{x, a}}\right)+\left(\frac{P_{x, a-1}}{N_{x, a-1}}\right)
$$

where $P=$ SCI population, $I=$ new SCI cases, $S R=$ survival rate, $N=$ years in age group, $x=$ current year and $a=$ age group.

For each year to be projected, the sum of the current year's estimated incidence and prevalence for a specific age cohort is adjusted for mortality by multiplying by the age specific survival rate. The surviving population is then reduced by the number of people who age into the next age cohort and increased by the number of people who enter from the preceding age cohort.

\section{Baseline prevalence}

Estimates of prevalence rates of SCI within the general population have been reviewed by Harvey et al $^{2}$ and range from 525 cases per million ${ }^{3}$ to 1124 cases per million. ${ }^{4} \mathrm{~A}$ recent national probability sampling study of the US SCI population that was conducted by Berkowitz et al $^{5}$ identified specific rates by gender and veteran status. These observations for the veteran population (1634 per million), male population (1050 per million), and female population (407 per million) were used to estimate a baseline SCI prevalence for each population segment.

Initial SCI prevalence was determined as the product of the 1988 prevalence rate and the total number of people in the population from which the SCI population originates (census estimates ${ }^{6,7,8}$ ). SCI population totals were then disaggregated and distributed into age groups according to percentages reported in the literature for the $1988 \mathrm{SCI}$ population (Table 1). Male and female age distributions that were used were those reported by Berkowitz et al, ${ }^{5}$ while the veteran age distribution that was applied was that observed by
Samsa et $a l^{9}$ in his mortality study of the veteran SCI population.

\section{Incidence}

Reported average incidence rates range from 29.4 cases per million ${ }^{10}$ to 50 cases per million. ${ }^{11}$ Most often cited are Kraus's ${ }^{12}$ estimate of 32.5 new cases of SCI admitted to hospitals annually (excludes DOA cases) per million population at risk, and Bracken's ${ }^{13}$ estimate of 40.1 per million. Both Kraus and Bracken have observed similar bimodal patterns of incidence. Rates peak between the ages of 16 and 25 and again in the group above 65. Male incidence rates are consistently higher than those for females, with male rates exceeding female rates on average 2.4 to $1 .^{13}$

Because of broad variation in age and gender specific incidence rates, an average rate could not validly be applied for the purposes of population forecasts. Age distribution of the population at risk is not stable over time, particularly within the veteran population which is skewed toward the upper age ranges as a result of historical recruitment patterns.

Another consideration in determining incidence to be applied in a model is that mortality rates during initial hospitalization are extremely high and age specific. ${ }^{12-14}$ Since the forecast model is designed to project the surviving population of SCI patients, incidence was calculated using age and gender specific post-hospitalization rates. These were calculated by reducing incidence rates to account for reported inhospital mortality. The rates for specific age groups used in the forecast model (Table 2) were calculated by interpolation of Bracken's published group values.

While higher than Kraus's rates, Bracken's incidence rates were used in the model because his study was of a broader scope than Kraus's, which was limited to 2 years of records and 18 California counties. Bracken's rates were derived from the National Hospital Discharge Survey database and included records from 1970 to 1977 , thus providing rates that are less likely to be subject to geographic or temporal influences. Notably, Bracken's rates were not significantly different from Kraus's for the 2 years covered in the California study and showed a slight trend of increasing rates over the 8

Table 11988 baseline SCI prevalence

\begin{tabular}{lrrrrr}
\hline $\begin{array}{l}\text { Age } \\
\text { Cohort }\end{array}$ & \multicolumn{2}{c}{ Veteran status } & \multicolumn{2}{c}{ Gender } & \multicolumn{2}{c}{ Total } \\
\cline { 2 - 5 } & Veteran & Non-vet & Male & Female \\
\hline $0-24$ & 1152 & 10593 & 7251 & 4494 & 11745 \\
$25-34$ & 6726 & 48827 & 38493 & 17060 & 55553 \\
$35-44$ & 13994 & 37581 & 41623 & 9952 & 51575 \\
$45-54$ & 8129 & 13471 & 14804 & 6796 & 21600 \\
$55-64$ & 9209 & 8605 & 71298 & 6516 & 17814 \\
$65-74$ & 5144 & 8431 & 4273 & 5658 & 13575 \\
$75+$ & 448 & 4387 & 125659 & 562 & 4835 \\
Total & 44802 & 131895 & 51038 & 176697 \\
\hline
\end{tabular}

aVeteran age range $18-24$ 
Table 2 PVA model incidence rates (per million)

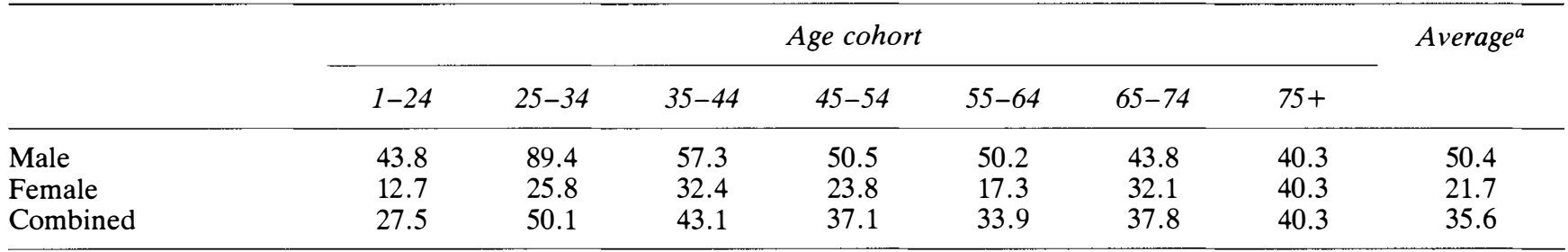

${ }^{a}$ Adjusted for age

year study period. Although Bracken's methodology has been criticized as possibly overestimating incidence, ${ }^{15}$ recent evidence from the New York and Oklahoma surveillance programs supports the use of Bracken's rates. ${ }^{16,17}$

Incidence for each projected year was estimated by multiplying the age and gender specific estimates of the population at risk by the appropriate incidence rate. Male rates were used for the veteran population since the number of females relative to the total veteran population was so small that using lower rates for female veterans would have an insignificant effect on the projected SCI population. The annual population at risk was calculated by subtracting the previous year's estimated SCI population (prevalence) from the total population estimate for each of the age cohorts identified in Table 2. The group specific incidence, calculated using the formula that follows, was then used to determine the SCI prevalence for the current year.

$$
I_{x, a}=\left(C_{x, a}-P_{x-1, a}\right) \times I R_{a}
$$

where $I=$ incidence, $C=$ census population, $P=$ prevalence, $I R=$ incidence rate, $x=$ current year and $a=$ age group

\section{Mortality}

The literature suggests that SCI survival rates should account for variability due to age at injury and injury classification. ${ }^{1,18,19}$ However, studies to date have not completely isolated the effects of age, age at injury, and injury class.

Published differences in mortality rates due to age at injury are confounded by the underlying effects of age, which is the predominant factor affecting the mortality rate. During the trauma care phase of treatment (initial 3 months), age at injury clearly is a significant factor determining mortality rate. ${ }^{12-14}$ However, mortality in the post traumatic care population follows a trend similar to the standard mortality of the non-SCI population $^{1,9,14,19}$ and must be considered separately from the incidence population when making estimates of mortality. Following the acute care phase, a close correlation exists between survival rates of the SCI population based on age at injury and standard survival rates based on age. Regression analysis of the logarithmic transformation of 1 year survival rates (derived from 10 year rates based on age at injury reported by the National Institute on Disability and Rehabilitation Research Model Spinal Cord Injury System (NIDRR
Model System $)^{1}$ ) resulted in an $r^{2}=0.988$. The variation in SCI survival between age groups relative to the variation in survival of a matched non-SCI population can be expressed with the following linear equation.

$$
\begin{aligned}
\log _{10} Y= & 1.47325489 \times \log _{10} X+(-0.951013) \\
& \pm 0.00475235
\end{aligned}
$$

where $Y=\mathrm{SCI}$ survival rate, $X=$ standard age, sex specific life table rate.

The relationship suggests that the change in the difference existing between survival rate of the general population and the SCI population reported by age at injury actually reflects the 'accelerated aging' process observed by researchers. ${ }^{1,9,19}$ The slope is characteristic of the relative difference due to age, and the $\mathrm{Y}$ intercept quantifies the initial impact of SCI on mortality rate. The equation represents the relative effect of SCI on mortality as age increases. By equating survival rate of the SCI population with the survival rate of the non-SCI population, published age and gender specific standard mortality rates of the population segment of interest can be used to estimate SCI survival rates. In this way differences in mortality rate due to gender are accounted for.

Krause $^{20}$ has observed that older people with SCI are more susceptible to physiological stress and infection, which ultimately is manifested in a higher mortality rate. The degree to which this applies to a person who has recently been injured as well as to one who has lived with SCI into old age, is uncertain. As DeVivo has noted, when young people age with SCI, it is not clear if they will experience the mortality rates associated with patients injured at ages above $50 .{ }^{18}$ In fact, the association between aging and declining health status in the SCI population has been observed to be similar to that of the general population. ${ }^{21}$ This suggests that the increase in susceptibility to physiological stress and infection over time is primarily due to age rather than duration of injury. Intuitively, one would expect that as time since injury increases, the relative significance of the effect of age-at-injury on mortality would decrease. Evidence supporting this hypothesis is seen in observations of long term SCI survivors reported by Whiteneck et al. ${ }^{19}$ The study showed that with increasing age the causes of death begin to approximate causes of death of the general population, regardless of severity of injury. Furthermore the relationship between mortality rates of long term SCI survivors and those of an age matched 
non-SCI population, approximate those described in cross sectional studies. ${ }^{1,9}$ Within this construct, survival rate of a particular age at injury group could be used as a measure of the survival probability for anyone of that age who has sustained traumatic SCI, regardless of how long the individual has been living with the injury.

The survival rates used in the model and provided in Table 3 were calculated from published standard rates of the US male and female population. ${ }^{22}$ Veteran rates were derived from standard white male rates since veterans were deemed to have a better initial overall health status than the general male population. ${ }^{9}$ It should be noted however, that in the uppermost age groups $(75+)$ the annual probability of survival for white males is less than that for the general male population. ${ }^{22}$

\section{Results}

The model's projections indicate a need for future expansion of capacity to treat traumatic SCI in the private sector, and a need to prepare for an aging disabled population. A $20 \%$ increase in the US SCI population can be expected over the next 10 years from approximately 207000 estimated in 1994, to 247000 . The ratio of approximately 2.3 males to each female will remain relatively stable. Females currently comprise $30 \%$ of the population with 61400 estimated.

In 1994 the veteran component of the SCI population represents almost $22 \%$ of the total and more than $35 \%$ of the SCI population aged 65 or more. A majority of SCI veterans are served by Veterans Health Administration (VHA) which has established itself as a national leader in the care and rehabilitation of people with SCI. Veteran demographics indicate a need to expand both institutional and non-institutional longterm-care services for this population. However, in the next 10 years, the veteran segment of the SCI population is projected to decline $16 \%$, while the non-veteran SCI population is expected to grow an estimated $27 \%$ (Figure 1). Within 20 years the veteran portion of the SCI population is expected to be reduced to $12 \%$. The result of these trends will be a shift of case load to the private sector that suggests a need for future redistribution of SCI health care resources.

The demand for SCI trauma care services in the future can be measured as a function of the annual number of traumatic SCI cases admitted to hospitals.

Table 3 Annual survival rates (SCI)

\begin{tabular}{lccc}
\hline Age range $^{a}$ & Female & Male & Veteran \\
\hline $1-24$ & 98.915 & 98.855 & 98.754 \\
$25-34$ & 98.862 & 98.668 & 98.708 \\
$35-34$ & 98.767 & 98.523 & 98.581 \\
$45-54$ & 98.483 & 98.093 & 98.172 \\
$55-64$ & 97.691 & 96.778 & 96.854 \\
$65-74$ & 96.063 & 94.110 & 94.172 \\
$75-84$ & 91.848 & 87.598 & 87.562 \\
$85+$ & 79.619 & 74.515 & 73.915 \\
\hline
\end{tabular}

a Veteran age range 18-24

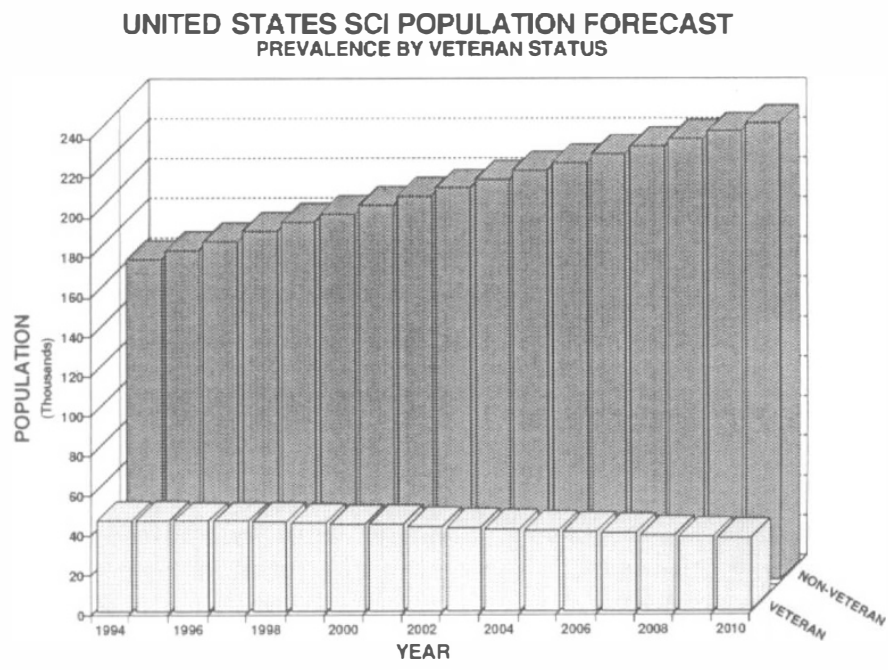

Figure 1 United States SCI population forecast: prevalence by veteran status

For this, the model predicts a gradual increase from approximately 11500 in 1994 to almost 13400 in 2010. Approximately $88 \%$ of these patients can be expected to be discharged and become part of the prevalence population (calculated by applying age and gender specific in-hospital mortality rates ${ }^{13}$ to the appropriate segments of the 1994 incidence population). The adjusted post-hospitalization incidence rate in 1994 is estimated at approximately 38 per million ( 23 per million for females and 55 per million for males).

The mean age of the SCI population is expected to increase in the coming decades as the general population ages and treatment protocols are improved. The number of people age 65 or more with SCI is estimated to be around $11 \%$ of the total SCI population in 1994 . By 2025 the proportion will grow over $24 \%$, with almost 73000 in this age group (Figure 2).

The model's predicted survival rate enables a midrange estimate of prevalence. This can be compared with values derived by applying survival rates representing the $95 \%$ confidence interval of predicted rates (Table 4). Since the algorithm that estimates projected SCI prevalence is recursive, the range of the estimate $(P<0.05)$ expands with each year from the baseline year 1988 (precision decreases).

Forecasts were also made using Kraus's incidence rates $^{12}$ to provide a comparative measure of the lower limit for prevalence. In 1994, differences between corresponding values are between $4 \%$ and $13 \%$ lower than those estimated with Bracken's rates, with the greatest deviation occurring in estimates of the female population.

\section{Discussion}

The model's strength lies in enabling population based estimates that account for age and gender composition, and providing a view of how the relative proportions between segments of the SCI population change over time. The model also may be used to establish a 
66

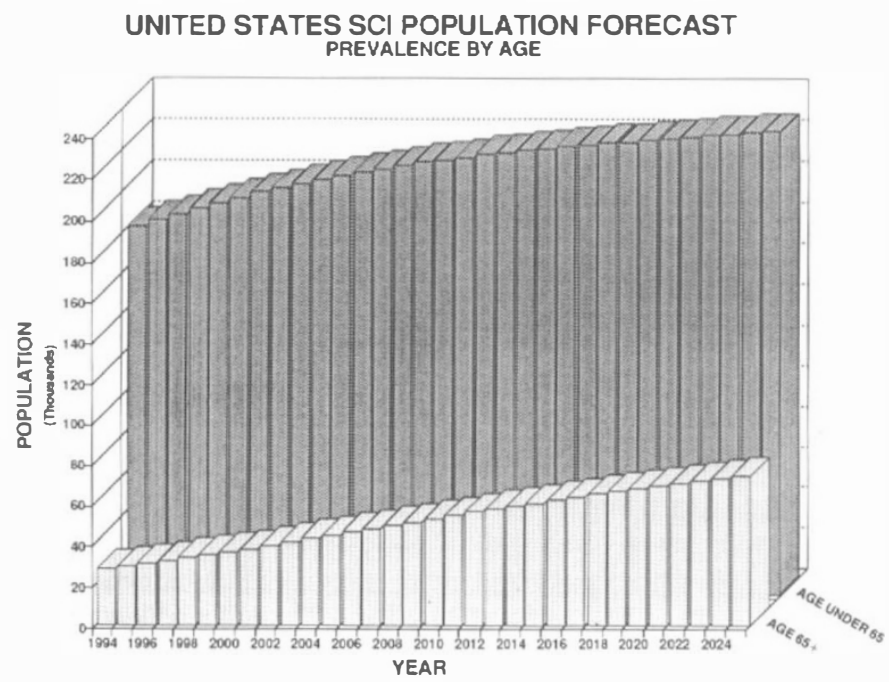

Figure 2 United States SCI population forecast: prevalence by age

benchmark against which state health agencies can gauge effectiveness of local SCI prevention efforts. It might even be used to enable states such as Arkansas, that provide case management services to their SCI citizens, to evaluate the capture rate achieved by their registry.

While the model has not been refined to the point of providing etiology specific estimates, overall incidence and prevalence can be obtained by applying the model to state census numbers. Since national rates are used, values represent the expected national norm for a particular state. Time trend analysis of the incidence rate observed by a state surveillance system relative to the expected norm before and after implementation of a prevention program would then control for the effects of changes in a population's age or gender structure.

Several assumptions were necessary to support the model because of the fragmented nature of available epidemiological data. Departure from these assumptions should be considered when interpreting model forecasts.
A basic underlying assumption is that the effect of differences in injury class case mix between age groups is reflected in the age specific survival rates reported for the NIDRR Model Systems population. ${ }^{1}$ The injury class composition of an age group is a function of the cumulative etiology of injury experienced by members of the cohort as it moves through time. Therefore changes in etiology specific incidence rates that have been observed in recent years ${ }^{23,24}$ will likely lead to gradual changes in case mix within individual age groups of the prevalence population. Furthermore, if improvements in survival rates are not uniform across neurological categories, survival within individual age groups will be affected differently. Nevertheless the predominant factor determining survival probability will be age, and while there may be minor changes in relative survival between age groups due to other factors, the overall variation is minimized by relating SCI survival to that of the general population.

Use of Model Systems survival rates also implies that the SCI population segment to be estimated will experience relative survival rates comparable to those of the Model Systems population. This will depend not only on injury class case mix but also on the quality of care that patients receive.

Comparison of population distributions on the basis of injury classification indicates that case mix of the Model Systems is skewed toward more severely injured patients who typically experience lower survival rates. ${ }^{5,21}$ Prevalence would therefore be likely to be underestimated by applying these rates to the general SCI population.

In the veteran population a significant shift can be expected over time, from a population characterized by a high percentage of less severely injured incomplete paraplegia, to one with a more even distribution. Because of this, survival of veterans is likely to be understated in the near term by using Model Systems rates. As newly injured veterans become a larger part of the total veteran SCI population, the injury class composition and therefore survival rate should approach that of the non-veteran population.

Confounding the effect of case mix variation is the

Table 4 Estimated prevalence of traumatic spinal cord injury in the US

\begin{tabular}{|c|c|c|c|c|c|c|}
\hline Group & Year & Predicted & High & Percent ${ }^{a}$ & Low & Percent ${ }^{a}$ \\
\hline \multirow{3}{*}{ Total } & 1994 & 207129 & 221275 & 6.8 & 196977 & 4.9 \\
\hline & 2004 & 246882 & 285442 & 15.6 & 221698 & 10.2 \\
\hline & 2014 & 276281 & 337095 & 22.0 & 239639 & 13.3 \\
\hline \multirow{3}{*}{ Male } & 1994 & 145763 & 156255 & 7.2 & 139075 & 4.6 \\
\hline & 2004 & 172840 & 201273 & 16.4 & 156376 & 9.5 \\
\hline & 2014 & 193415 & 238102 & 23.1 & 169598 & 12.3 \\
\hline \multirow{3}{*}{ Female } & 1994 & 61366 & 65050 & 6.0 & 57902 & 5.6 \\
\hline & 2004 & 74042 & 84205 & 13.7 & 65322 & 11.8 \\
\hline & 2014 & 82866 & 98993 & 19.5 & 70041 & 15.5 \\
\hline \multirow[t]{3}{*}{ Veteran } & 1994 & 45626 & 48432 & 6.2 & 42883 & 6.0 \\
\hline & 2004 & 40950 & 47187 & 15.2 & 35473 & 13.3 \\
\hline & 2014 & 33055 & 40630 & 22.9 & 26975 & 18.4 \\
\hline
\end{tabular}

aPercent deviation from predicted value 
difference in the quality of care and access to a continuum of required services. Evidence suggests that the Model SCI Systems provide superior SCI care. ${ }^{25,26}$ This being the case, survival rates would probably be overstated for the general SCI population by applying Model Systems rates. The disparity should not be as great for the veteran population since the specialized SCI rehabilitation and follow-up services provided by the VA more closely approximate those of the Model Systems.

The bias introduced to national prevalence estimates by using Model Systems survival rates would be minimized by the offsetting influences of superior care and a more severely injured case mix. While the net effect cannot be measured, it is likely that it is not significant enough to cause deviation beyond the $95 \%$ range of certainty projected by the model.

Finally, use of Bracken's incidence rates implies an assumption that incidence rates are stable and have not changed significantly in the last 15 years. The model shows that even with fixed age and gender specific incidence rates, the overall incidence rate will increase slightly due to changes in age distribution within the population. This could account for the increasing trend noted by Bracken. ${ }^{13}$ However, factors such as improvements in medical technology can be expected to lead to survival beyond the $88.8 \%$ observed by Bracken in the 1970s hospitalized incidence population, and better prognosis following initial rehabilitation. This improvement may be offset by a greater proportion of new injuries occurring to older people as a consequence of the increasing mean age of the general population. Since these age groups generally experience higher mortality during initial hospitalization, ${ }^{12,13}$ net improvement in survival rates of the hospitalized incidence population may, in fact, be minimal.

The apparent inconsistency of a $93.7 \%$ survival rate reported by the Model Systems for day 1 admits in the first year following injury ${ }^{18}$ may be explained for the most part by the fact that deaths occurring within the first $24 \mathrm{~h}$ following injury were excluded. Kraus's seminal study on incidence of SCI showed that $3.9 \%$ of the cases admitted to hospitals died within the first $24 \mathrm{~h}$ following surgery. ${ }^{27}$ If this value is used to adjust the Model Systems rate to equate it with Bracken's NHDS derived rate, that includes hospitalized deaths within the first $24 \mathrm{~h}$, the difference is negligible.

Further indication that Bracken's rates would not bias estimates is found in more current population based estimates of incidence ${ }^{11}$ and reports from some state surveillance systems. ${ }^{16,17}$

\section{Conclusions}

It is clear that updated incidence rates and more current age and gender specific baseline prevalence estimates would enhance the utility of the model. The accuracy of incidence rates can be expected to be improved with the evolution of the Center for Disease Control and Prevention's state SCI surveillance system $^{28}$ and more extensive use of E-codes (external cause of injury codes) by admitting hospitals. ${ }^{16}$ If more states follow the lead of Arkansas and develop a registry of their SCI citizens, they could, in aggregate, provide a reliable proxy for calculating national prevalence rates. In October of 1994, VHA is scheduled to launch a national spinal cord dysfunction registry of all veterans with either traumatic or non-traumatic injury. Its success will provide a valuable resource for longitudinal studies of outcomes and aging with SCI. In addition, longitudinal studies of SCI populations, such as those being conducted by the Model System projects, are beginning to provide the mortality data necessary for more accurate long range projections. Future modifications to improve the forecasting model might also include: age groupings that are smaller than the 10 year cohorts that were used, and modeling each injury class separately, with classifications divided according to life expectancy groupings reported by DeVivo at the 1994 annual American Spinal Injury Association (ASIA) conference. ${ }^{24}$

As it stands, the model provides a relatively robust method for estimating and forecasting both incidence and prevalence of traumatic spinal cord injury within the US and it provides a framework into which updated population specific data may be incorporated as it becomes available.

\section{References}

1 Stover S, Fine PR. Spinal Cord Injury: The Facts and Figures. University of Alabama at Birmingham: Birmingham (AL), 1986.

2 Harvey C, Rothschild BB, Ashmann AJ, Stripling T. New estimates of traumatic SCI prevalence: A survey based approach. Paraplegia 1990; 28: 537-544.

3 Kurtzke JF. Epidemiology of spinal cord injury. Exp Neurol 1975; 48: 163-236.

4 Collins JG. Types of injuries and impairments due to injuries. Vital and Health Statistics. Series 10, No. 159. DHHS Pub. 87-1587, 1987.

5 Berkowitz M, Harvey C, Greene CG, Wilson SE. The Economic Consequences of Traumatic Spinal Cord Injury. Demos Publications: New York, 1992.

6 Day JC. Population Projections of the United States, by Age, Sex, Race and Hispanic Origin: 1992 to 2050. Bureau of the Census Pub. P25-1092, 1993.

7 Heltman LR, Bonczar TP. Estimates and Projections of the Veteran Population: 1980 to 2040. Department of Veterans Affairs, Pub. No. M-004-90-01, 1990.

8 Sorensen KA, Feild TC. Projections of the U.S Veteran Population: 1990-2010. Department of Veterans Affairs, Pub. No. SB-008-94-3, 1994.

9 Samsa GP, Patrick CH, Feussner JR. Long-term survival of veterans with traumatic spinal cord injury. Arch Neurol 1993; 50: $909-914$

10 Fine PR, Kuhlemeier KV, DeVivo MJ, Stover S. Spinal cord injury: An epidemiologic perspective. Paraplegia 1979; 17: 237-250.

11 Ergas, Z. Spinal cord injury in the United States: A statistical update. Cent Nerv Syst Trauma 1985; 2: 19-30.

12 Kraus JF et al. Incidence of traumatic spinal cord lesions. J Chron Dis 1975; 28: 471-492.

13 Bracken MB, Freeman DH, Hellenbrand K (1981) Incidence of acute traumatic spinal cord injury in the United States, 1970-1977. Am J Epidemiol 1981; 113: 615-622.

14 Mesard L, Carmody A, Mannarino E, Ruge D. Survival after spinal cord treatment: A life table analysis. Arch Neurol 1978; 35: $78-83$ 
15 Fine PR, DeVivo MJ, McEachran AB. Incidence of acute traumatic hospitalized spinal cord injury in the United States, 1970-1977. (Letter to the Editor). Am J Epidemiol 1982; 115: 475-479.

16 Relethford JH, Standfast SJ, Morse DL. Trends in traumatic spinal cord injury-New York, 1982-1988. MMWR 1991; 40: 535-537, 543.

17 Price C, Makintubee S, Herndon W, Istre GR. Epidemiology of traumatic spinal cord injury and acute hospitalization and rehabilitation charges for spinal cord injuries in Oklahoma, 1988-1990. Am J Epidemiol 1994; 139: 37-47.

18 DeVivo MJ, Stover S, Black KJ. Prognostic factors for 12-year survival after spinal cord injury. Arch Phys Med Rehabil 1992; 73: $156-162$.

19 Whiteneck GG et al. Mortality, morbidity, and psychosocial outcomes of persons spinal cord injured more than 20 years ago. Paraplegia 1992; 30: 617-630.

20 Krause JS, Crewe NM. Chronologic age, time since injury, and time of measurement: Effect on adjustment after spinal cord injury. Arch Phys Med Rehabil 1991; 72: 91-100.
21 DeVivo MJ et al. A cross-sectional study of the relationship between age and current health status for persons with spinal cord injuries. Paraplegia 1992; 30: 820-827.

22 Statistical Abstract of the United States: 1993, 113th edn. US Bureau of the Census: Washington (DC), 1993.

23 DeVivo MJ et al. Trends in spinal cord injury demographics and treatment outcomes between 1973 and 1986. Arch Phys Med Rehabil 1992; 73: 424-430.

24 The National SCI Statistical Center. Spinal Cord Injury: Facts and Figures at a Glance. University of Alabama: Alabama, 1994.

25 DeVivo MJ et al. Benefits of early admission to an organised spinal cord injury care system. Paraplegia 1990; 28: 545-555.

26 Heineman AW et al. Functional outcome following spinal cord injury: a comparison of specialized spinal cord injury $v s$ general hospital short term care. Arch Neurol 1989; 46: 1098-1102.

27 Kraus JF, Franti CE, Borhani NO, Riggins RS. Survival with acute spinal-cord injury. J Chron Dis 1979; 32: 269-283.

28 Harrison CL, Dijkers M. Spinal cord injury surveillance in the United States: An overview. Paraplegia 1991; 29: 233-246. 